\title{
Pan FGFR Inhibitor PRN1371
}

National Cancer Institute

\section{Source}

National Cancer Institute. pan FGFR Inhibitor PRN1371. NCI Thesaurus. Code C124931.

A highly specific covalent inhibitor of human fibroblast growth factor receptor types 1, 2, 3 and 4 (FGFR1-4) with potential antiang iogenic and antineoplastic activities. FGFR1-4 tyrosine kinase inhibitor PRN1371 specifically binds to a conserved cysteine residue in the glycine-rich loop in FGFRs and inhibits their tyrosine kinase activity, which may result in the inhibition of both tumor angiogenesis and tumor cell proliferation, and the induction of tumor cell death. FGFRs are a family of receptor tyrosine kinases, which may be upregulated in various tumor cell types and may be involved in tumor cell differentiation, proliferation and survival, and in tumor angiogenesis. This agent potently inhibits FGFR1-4 but does not inhibit other tyrosine kinases, even those that share the conserved cysteine, which may improve therapeutic responses and decrease toxicity when compared with less selective inhibitors. 